\title{
Position Paper: The Usefulness of Data-driven, Intelligent Agent- Based Modelling for Transport Infrastructure Management
}

\author{
Olusola. T. Faboya ${ }^{1}$ (Student Member), Grazziela P. Figueredo ${ }^{1}$, Brendan Ryan ${ }^{2}$, Peer-Olaf Siebers ${ }^{1}$ \\ ${ }^{1}$ School of Computer Science, University of Nottingham, Nottingham, United Kingdom \\ ${ }^{2}$ Faculty of Engineering, University of Nottingham, Nottingham, United Kingdom \\ itxotfa,pmzgf,epzbr pszps @ nottingham.ac.uk
}

\begin{abstract}
The uneven utilisation of modes of transport has a big impact on traffic in transport pathway infrastrutures. For motor vehicles for instance, this situation explains rapid road deterioration and the large amounts of money invested in maintenance and development due to overuse. There are many approaches to managing this problem; however, the impact of individual users in infrastructural maintenance is mostly ignored. In this position paper, we hypothesise that important changes torwards a more efficient use of the transport network start with its users and their behavioural changes. To this end, we introduce our vision on how to employ data driven, intelligent agent-based modelling, incorporating human factors aspects, as a toolset to understand travellers and to stimulate behavioural changes. The aim is to achieve better balanced and integrated mobility usage within the transport network. The idea is explored with a few guided questions, and a methodology is proposed. We employ 1) cognitive work analysis to investigate the reasons for travellers' mode choice; 2) computational intelligence to extract and represent knowledge from related datasets; 3 ) agent-based modelling to represent the real-world and to observe both individual and emergent behaviours. Future directions to adapt our methodology to alternative smart mobility projects are also discussed.
\end{abstract}

\section{INTRODUCTION}

Globally, transport infrastructure is one of the key set of assets enabling the development of the economy. Nowadays, billions of dollars are being invested in its development and maintenance in response to increased demands as a result of population growth and the needs for mobility. Apart from the huge costs, the rapid growth in demand and maintenance of transport networks brings threats to the environment, economy, safety [1],[2], and social life. Transport infrastructure management has been mostly focusing efforts on minimising maintenance [3],[4] and development costs [5], while keeping safety, availability and reliability at acceptable levels. Achieving these objectives, however, is becoming increasingly challenging due to the shrinking global financial status, the ageing and subsequent deterioration of infrastructures [3] due to heavy usage. More importantly is also the fact that the roles of transport users in infrastructural maintenance is often ignored. All these factors combined impact heavily on the health and lifecycle of transport networks and should be considered by the stakeholders during decision making. Our interest is to propose ways of incorporating human factors, human behaviour and computational intelligence into simulation tools to help promote societal changes that positively impact on transport infrastructure health. The transport system is a sociotechnical system with people as one of its essential components. Regarding transport demand, people tend to choose a transport mode that (i) answers their mobility needs; (ii) is easy to use; (iii) is reliable and safe; and (iv) ensures access to markets and resources [6]. Travellers' mode choice decision processes are complex, depending on many interdependent factors, most of which are not crisp, but uncertain/fuzzy and subjective to the decision maker [7]. Individuals' preferences in mode choice over time have resulted in an uneven usage of available transport modes [8],[9], which make some infrastructures being under pressure due to heavy usage, and others underutilised. In order to tackle this problem, detailed investigations and surveys into users' operations within the transport system is essential, because decision-makers need to understand why individuals choose a travel mode over another [10]. In addition, it is important to identify what effects those choices have on the transport infrastructure lifecycle and on users' safety and economy. It is also necessary to understand how intervention to promote changes in users' behaviour can improve the health and life of transport infrastructures. Consequently, a few questions arise: How can reliable data be obtained to provide insights into the activities of the heterogeneous transport users? How can this information be incorporated into a simulation system for decision making? Are there adaptive intelligent systems to extract information from the large interdependent data stream constantly gathered from users and their user-transport infrastructural survey? How can uncertainty in data due to human nature and structural relationship be addressed? Our position is that the questions raised can be tackled using data-driven agent-based computational intelligence modelling approaches. This position paper therefore proposes an intelligent travel mode 
integration methodology that relies on cognitive work analysis, computational intelligence techniques, agent-based modelling and data mining algorithms to achieve a datadriven intelligent integrated mobility decision tool set to promote good health of transport infrastructure. Integration in transport mode involves interoperability between travel modes and within transport systems. Transport users are autonomous entities who have habits, interact, make intelligent decisions and adapt to a changing complex transport system's environment. Consequently, our approach investigates the dynamic transport environment using a Human Factors analytical framework called the cognitive work analysis [11],[12] with a view to identifying the constraints the system imposes on users. We also explore knowledge discovery with appropriate data mining algorithms to understand patterns emerging from the system; as well as a fuzzy logic systems to capture uncertainty in data imprecision. Also to model populations of heterogeneous, autonomous travellers that have behaviour and ability to interact with other travellers within the system using agentbased modelling technique. The individual traveller is created as an agent equipped with rules of behaviour extracted from the real-world data and simulated within the agent-based environment. The final objective is to obtain a system where it is possible to observe the emergent behaviour arising from the interacting entities within a simulation environment and their possible responses to new travel policies. We hope that the emerging behaviour of the simulation systems assisted with the knowledge unveil by the computational techniques, our methodology can provide information to aid efficient strategic development for mode integration that can lead to improvement in the health of the transport infrastructure.

\section{BACKGROUND INFORMATION}

Transport infrastructure includes physical networks, terminals and intermodal nodes, information systems and refuelling and electrical supply networks [13]. The investments in transport infrastructure across the world are on the rise[14][8] [15]. Available data show that road and rail infrastructure attract large investments [15] [8]. Despite the considerable investment in rail infrastructure, $73 \%$ of domestic freight moved in Great Britain in 2014, for instance, was done by road through heavy goods vehicles (HGVs); and this percentage increased to $76 \%$ in 2015 [9],[16]. Furthermore, a larger percentage of passenger mobility in the UK is done by road. Car use accounts for $78 \%$ of total distance travelled either as a driver or a passenger in 2014 [9]. The situation is not different in the US. Trucks have the largest shares of freight by values, ton, and ton-miles for shipment moved up to 750 miles [8]. Also, nearly four-fifths of person-miles of travel (PMT) was in cars or other personal vehicles while domestic air travel accounted for $11 \%$.

It is therefore evident that some transport modes attract more freights and passengers' mobility than the others. It is also clear that there are uneven distributions in the shares of services across the travel modes. Hence, this has made some modes such as road transport more problematic regarding infrastructural usage, traffic congestions, safety, reduced economy and its overall infrastructure health. There are several approaches to mitigate the many challenges of the transport system regarding mode choice and better use of the infrastructure. Among the possible solutions is integrated mobility or the travel mode integration approach. Although mode integration was suggested to curb greenhouse gas emissions (GHG) [13], with the appropriate deployment of specialised knowledge gathering innovations and data-driven computational techniques, it can be used to resolve issues of serious concern such as infrastructural deterioration due to accumulated effects of uneven travel mode distributions. Consequently, an in-depth understanding of transport system's entities and their interactions is essential to enhance proper strategic policies formulation to propel necessary changes. But access to accurate and complete datasets and proper harnesses of relevant methodologies to support knowledge discovery is necessary for the achievement of efficient transport infrastructure management through mode integration.

\section{A. Sociotechnical Transport System}

The sociotechnical theory is based on the idea that the design and performance of an organisational system can only be understood and improved if both 'social' and 'technical' aspects are brought together and are treated as interdependent parts of a complex system [17]. The social aspect of the transport domain as a sociotechnical system comprises, among other things, of heterogeneous human beings with behaviour and capabilities to adapt to changes in the environment. But the technical systems might not be perfect and, as such, not able to cope with the demands of changing environments in which human are situated [18], that explains changes in humans' reactions when a situation needs improvement or when things go wrong in a system [10]. Human activities can be understood in terms of constraints that restrict them, and the goals that provide direction to their actions, procedures and decision. It is therefore important to understand the performance-shaping factors of the domain within which users' operations are performed. Such understanding according to Bisantz et al. [19] will not only be helpful but will also be necessary to make sense of and support performance in complex, unpredictable environments. Consequently, Human Factors (HF) investigates factors and the development of tools that facilitate the achievements of enhancing performance, increasing safety and increasing user satisfaction [20]. The application of HF analytical techniques is key to understanding the factors that shape users' travel mode choices.

\section{B. Human Factors approaches to complex system analysis}

There are several analytical techniques in Human Factors. The methods include normative task analysis that prescribes how a system should behave [12],[21]; there is also the descriptive task analysis [22],[19] that focus on analyzing how a system behaves in practice. The two techniques work by decomposing activities into a set of task sequences, which can rarely be extended beyond stable and repeatable systems [23]. Thus, those techniques are not suitable for unanticipated 
events that occur in a dynamic transport environment. Consequently, the formative approach has the capabilities to handle inherent complexity and adaptability. It also allows the examination of unpredicted, and unanticipated actions within a system. Cognitive Work Analysis (CWA) [11],[12] is a well-established formative task analysis model that had been used in Human Factors and Ergonomics to investigate the constraints imposed on the users by the system's environments.

CWA is a five-phase model that offers deep insight into the work analysis of complex systems by defining what is needed to perform the task, regardless of the actors, the situation and the environment of the system. Details of CWA can be found in [12],[24]. It has been applied successfully in many domains such as process control [12], transport [25],[26]. This paper discusses only the first phase of CWA, i.e. work domain analysis (WDA) which is relevant to the proposed methodology. The WDA defines the reasons for behaviour within the system (functional properties) and defines the resources available for the behaviour (physical properties) [22]. WDA models system with the Abstraction Hierarchy (AH) which uses 'how-what-why' triads to describe the relationship among the elements within the system domain across five conceptual levels in the WDA. An illustrative example of how AH works is given in Section III (step 4). The emergent behaviours arising from individual autonomous traveller is key to understanding the system's response to interventions. The next section discusses the modelling of individuals within a complex adaptive system.

\section{Agent-Based Modelling and Travellers' Heterogeneity}

Beanland et al. [27] describe agent-based modelling (ABM) as a way to model the dynamics of complex systems and complex adaptive systems. It models a system from the perspective of agents, i.e., viewing any system as consisting of agents. Agents are entities within a system that have behaviours, can interact with other entities and the environment. Their interactions with other entities can influence their behaviours [28]. Individual behaviour and their interactions are explicitly represented in a program or even in another physical entity such as robot [29]. Such agent is capable of changing its behaviour during the simulation in an adaptive system as agents learn, encounter novel situations, or as populations adjust their composition to include larger proportion of agents who have successfully adapted [30]. However, to mimic the behaviour of humans they represent, agents require to be equipped with the right, and adequate set of rules developed from extracted real-life data representing the observed population. The techniques for abstracting knowledge from datasets are reviewed in the following section.

\section{Computational Intelligence Techniques}

The nature of transport system as a sociotechnical system, and the heterogeneity in travellers' attributes, as well as possible uncertainties that can arise in their mode choice decision process, require computational techniques that can perform well in the complex and adaptive system environment. Such techniques are necessary to handle the huge volumes of interdependent data arising from human activities and operations within the transport domain. The objective is to assist understanding and to mimick 'intelligent behaviour'. Engelbrecht [31] describes Computational intelligence (CI) as the study of adaptive mechanisms to enable or facilitate intelligent behaviour in complex and changing environments. Any methodology that is capable of assisting computers to behave intelligently in addressing complex world problems involving large and interdependent data, as well as imprecision and uncertainty information is part of CI. For our methodology, within CI we include the use fuzzy logic systems (FLS) [32]. They concern with how people describe the world around them [33] and represents decisions that are rather ambiguous and blurred [32]. In practice, travellers have in mind the vague idea of their preference in travel mode with a multitude of attributes and factors that have no distinct boundaries; such complex situations are often best handled with the application of the fuzzy system. These systems will be used to mimick travellers decisions. Evolutionary computation [34] and the swarm intelligence [31] can assist optimising parameters, modelling social networking among the agents (travellers) and also selecting neighbour agents to engage in interaction. Furthermore, machine learning methods will assist extracting data patterns, clustering behavior and predicting classes or outcomes. CI techniques will therefore provide the support to extract and incorporate knowledge from interdependent datasets into agents and their environment.

The next section describes the processes involved in the methodology to support transport infrastructure management through mode integration.

\section{PROPOSED SOLUTION}

This section describes our proposed solution by bringing together various modelling techniques earlier discussed. Illustrative example of freight mobility from the origin-todestination is used to work through the stages in the methodology process.

The process flow diagram depicted in Figure 1 provides a guide to understanding how the methodology can be used to promote transport infrastructure through mode integration. Step 1 of the process flow is the starting point.

Step 2 is to investigate what constitutes the reasons for the choice that people make when selecting a mode for their freight mobility. Knowledge gathering methods such as interviews, questionnaires, document analysis etc., in addition to specialized vehicular data collection devices, such as video vehicle detection, pneumatic road tube counting, piezoelectric sensor that measure vehicular flows and weights can be used. The focus of the data gathering should reflect the reasons for the preferences in a mode and people's perceptions on the available existing infrastructure that support their journey, as well as their likely responses to interventions if provided.

Step 3 focuses on the exploration of collected data to extract and identify infrastructures, procedures etc., within the system that are of concern of the participants. The data 
exploration will assist the construction of the abstraction hierarchy in step 4 in order to describe the relationships that exist within the system.

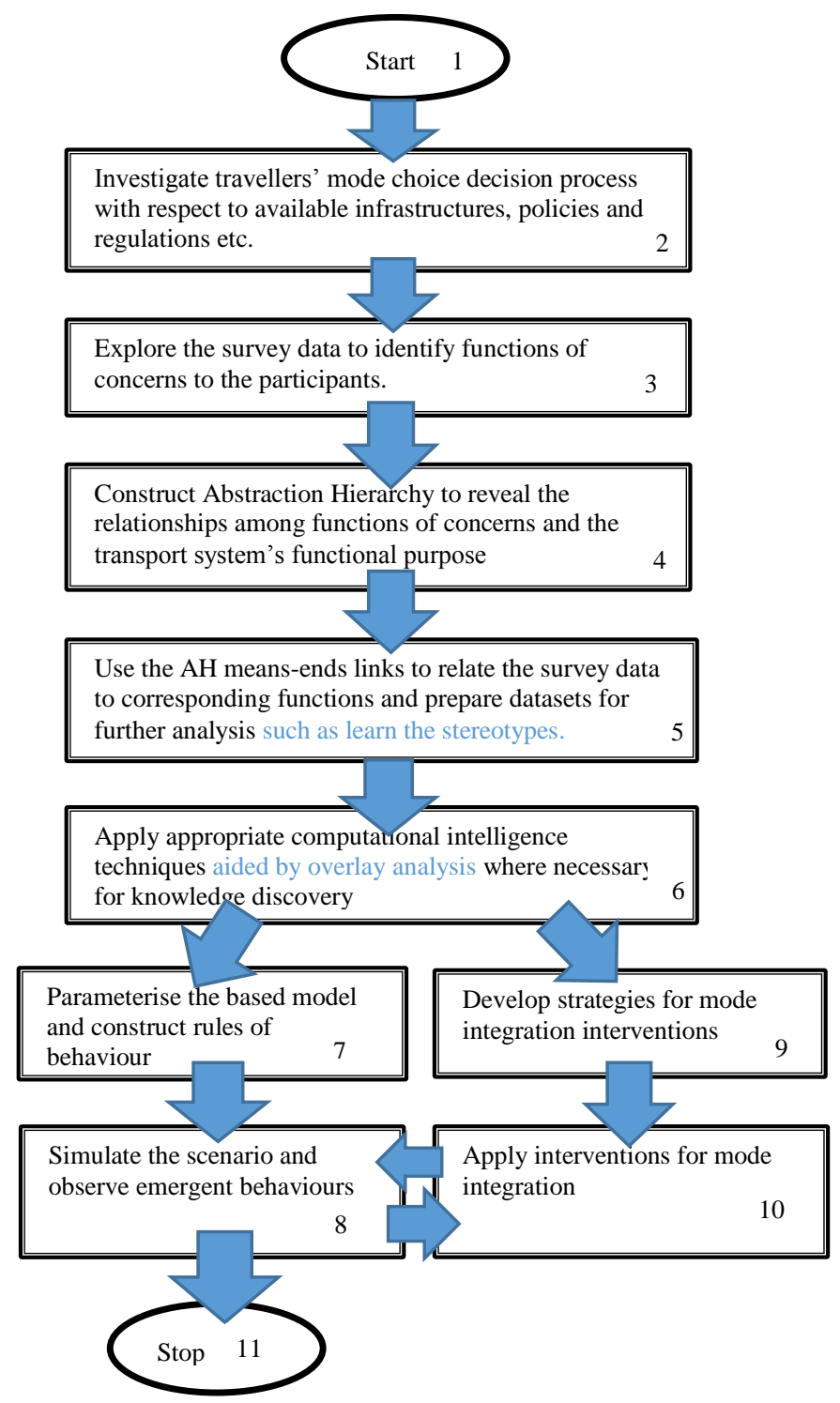

Figure 1. Mode Integration Methodology Process Flow

Step 4 is inspired by [35] and thus explained with the aid of the AH in Figure 2 which shows our vision of infrastructure and resources that support travellers' activities to achieve the purpose of goods delivery to the destination.

Starting from the top level (see Figure 2), the Functional purpose (top-left box on level 1 of Figure 2) of a transport system is to provide necessary infrastructural support for a comfortable and efficient movement of goods from the origin to the destination. The level below the functional purpose level (level 2) is the Values and priority measures that have Reliability, Journey time and Cost and value for money as the criteria defined to evaluate how the system progresses towards its functional purposes. For instance, moving goods from origin to destination can be impacted by route networks hence, prompt the traveler to show preference for alternative travel modes that has better network connections. The middle level (level 3) is the Purpose-related functions, which describe the general functions necessary for a system to achieve the functional purpose. Among the general functions for our example are cater for tasks needs, which refers to the system capability to provide necessary support for freight transfer functions; origin-destination connections which include efficient routes connection of origin-depotsdestination; information provision that can enhance smooth travels; and drivers and freight protections for general safety within the system, all are to assist the achievement of efficient and comfortable freight movement. The Object-related processes (level 4) refer to the functional capabilities and limitations of the current objects and infrastructural resources within the system which affect achievements of the efficient and comfortable freight delivery, and lastly, the Physical objects and Resources (level 5) which consist of the actual available infrastructural objects and resources within the system that the object-related processes refer. The AH nodes are connected by the means-ends links that describe the relationships among the boxes such that when a node is taken within the hierarchy as the 'what', nodes linked in the hierarchical level above the node indicate 'why' the chosen node is necessary within the system. Any connected nodes on the level immediately below that node can be taken to answer the question of 'how' that function is to be achieved or fulfilled [12]. For example, tracing through the highlighted links and boxes in Figure 2, if 'cater for task needs' (box 3 in level 3) is taken as 'what' at the purpose related functions level. The links connecting the node up to the value and priority measures level show that it can support 'cost and value for money' node. i.e. it occurs to forms part of the considerations to evaluate the costs of moving the good to the destination as well as the value for money (i.e. why). Also, to show how 'cater for task needs' (what) has been derived. The level below i.e. the object related processes level that has 'route/networks connections', 'wayfinding', 'general border processes', and 'energy supply' highlighted (i.e. how) showing how the cater for needs node was derived.

In step 5, the information provided by the AH components' relationships will be used to organize the survey data with the related functions they support. Then the data will further be analyzed for travellers' stereotypes elucidation using clustering algorithms such as K-Means. CI techniques extract useful information from the survey data. These techniques will involve the use of overlay analysis due to possible differences in the formats of the collected survey data. 1) Data collected from both human and vehicles will require fusion; in addition, different sources will require weighting factors based on their importance to the overall objective of the system. 2) Correlation analysis of the multiple sources of data will establish the relationship of all inputs before combining the data for further pattern identification. 3) the challenges of the uncertainties and imprecision in the data are better handled with a Type-1 fuzzy logic by using precise membership functions describing the agents decision based on the data analysis and stereotypes defined previously [36]. 


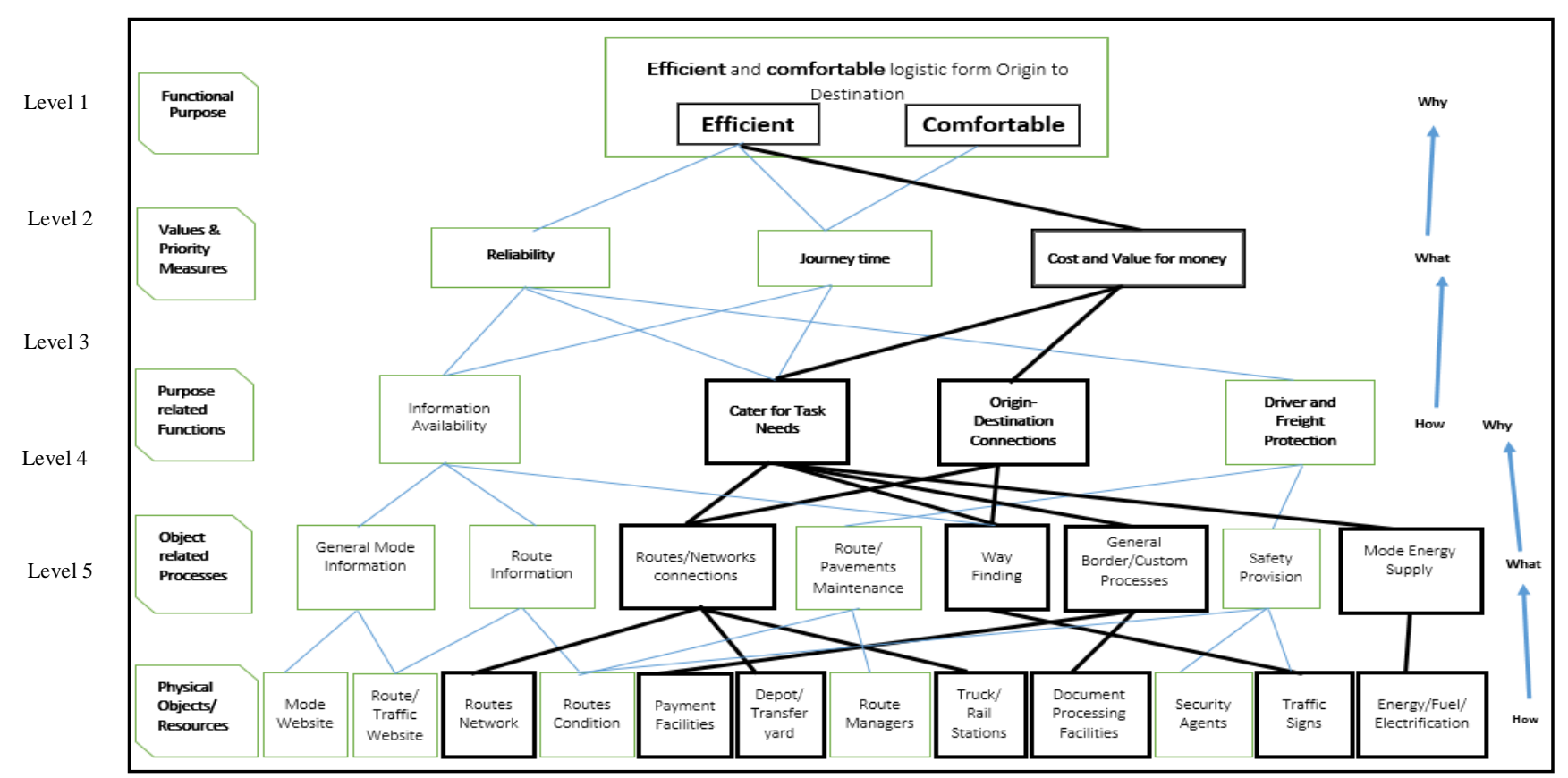

Figure 2. The Freight Mobility Abstraction Hierarchy (Inspired by [35]

Duch [37] argues that an important challenge for CI is to create flexible systems that can use their modules to explore various ways to solve a given problem, proposing a different solution that may have different advantages. With agentbased simulation, each of the agents (virtual traveller) in the system will be preloaded with rules of behaviour which often are the products of series of analysis carried out in step 6.

In step 7, the agents' stereotypes are calibrated accordingly using the information from the previous analysis, and the model is simulated in step 8. Based on the parameterisations in step 7, the simulated settings represent the system base scenario with which further experimentation can be compared. The knowledge gained from the $\mathrm{CI}$ applications in step 6 will also provide support for strategic interventions development in step 9, which is for the purpose of stimulating travellers behaviours towards the reduction in the share of road transport in freight mobility. In step 10, the interventions can be applied to the already known base scenario agents' behaviours (i.e. step 8). Several experimentation of such interventions can be performed, and individual autonomous agent's behaviour, as well as the aggregate emergent behaviours, can be observed to better understand travellers' behaviour in response the interventions.

\section{DisCUSSIONS AND FUTURE DiRECTIONS}

Effective mode integration through appropriate interventions to stimulate travellers mode choice behaviour positively impact the whole transport infrastructure lifecycle. It reduces for instance development time, maintenance, costs, emmissions and violation of land use acts. However, the objective of achieving travel mode integration could be difficult to accomplish within the current transport policies operations in many countries. For instance, in some countries including the UK, freight mobility services are autonomous, stand-alone, and information about goods movement are preserved within each company [38], while the infrastructures are owned by both private and public organisations. In the future, there is a need for comprehensive information sharing among transport companies for the integration of larger regional and continental mobility systems so as to promote sustainable and efficient linkages among infrastructures and other facilities that include all travel modes. Also, policies and regulations that encourage collaborative transport solutions should be put in place.

Currently, transport users are embracing digitalisation with the use of web applications, smartphones, social media etc. These new lifestyles can be explored further in the future to promote and improve passengers' journey experience, tailored to their individual needs and preferences. Furthermore, actions such as promoting public awareness about the needs for modal integration, providing information about various mode services and infrastructures such as route networks, intermodal terminals, park and ride services, transfer yards, and depots for goods can potentially have significant transformative effects on passenger's behaviour. Such information can be embedded within smart devices to enable door-to-door smart mobility and increase resources efficient utilisation. The need to increase integrated management of services such as integrated ticketing and document processing system, real-time information that cover all modes, will encourage mobility across all travel modes.

\section{CONCLUSION}

A proposed methodology to promote transport infrastructure health and lifecycle through travel mode integration was introduced in this paper. There has been an increase in the mobility demand and increasing share of road travel modes while other modes were underutilised for freight and passenger mobility. The situation has impacted negatively 
on the health of road infrastructures. However, to achieve mode integration, decision-makers need to understand why individuals choose a travel mode over another, and also, they need to identify what effects those choices have on transport infrastructure lifecycle and on users' economy. It is also important to understand how stimulating changes in users' behaviour can improve the health and life of transport infrastructures. The structure and activities within the transport system environment are both complex and consist of elements that exhibit adaptive behaviours. Hence, useful ideas from related branch of disciplines such as CWA, ABM and CI that deal with complex adaptive systems are carefully investigated and harnessed to provide answers to the issues raised. A methodology was developed with each of its components addressing a part of the concerns as follows: the inclusion of Human Factors CWA and its WDA abstraction hierarchy provided investigative opportunity to answer the question 'How can reliable data be obtained to provide insights into the activities of the heterogeneous transport users?'. Features extraction methods and agent-based modelling techniques supported with the rules of behaviour generated through the application of appropriate computational intelligence knowledge which are necessary to observe agents' behaviour will explain 'How can this information be incorporated into a system for decision making?'. The integration of CI techniques into the methodology process for knowledge extraction provide the needed technical support that answers the question 'Is there any technical supporting system with an adaptive mechanism to obtain useful information from the vast non-linear data constantly gathered from users and the user-transport infrastructural survey? The Fuzzy logic system as a CI technique is an established tool for dealing with uncertainty in data imprecisions which could be a major challenge in representing travellers' perception. The fuzzy system incorporation provided solutions to 'How can uncertainty in data due to human nature and structural relationship be addressed?' Lastly, due to the trend in the smart mobility projects ongoing globally. Some future directions of making mode integration method to be continually relevant to impact on infrastructural health and lifecycle are suggested.

\section{REFERENCES}

[1] A. Griškevičiūtè-Gečienè and D. Griškevičienè, "The Influence of Transport Infrastructure Development on Sustainable Living Environment in Lithuania," Procedia Eng., vol. 134, pp. 215-223, 2016.

[2] G. P. Figueredo, et al., "Multi-Objective Optimization for Preventive Maintenance in Transportation: A Review," Tech. Rep. (2018)

[3] G. Kabir, R. Sadiq, and S. Tesfamariam, "A review of multi-criteria decision-making methods for infrastructure management," Struct. Infrastruct. Eng., vol. 10, 2014.

[4] J. Selih, et al., "Multiple-Criteria Decision Support System in Highway Infrastructure Management," Transport, 23, no. 4, pp. 299-305, 2008.

[5] H. Abou-Senna, et al., "Integrating transportation systems management and operations into the project life cycle from planning to construction: A synthesis of best practices," J. T Transp. Eng., 5, 1, pp. 44-55, 2018.

[6] J.-P. Rodrigue, et al., The Geography of Transport Systems, 4th ed. New York: Routledge, 2017.

[7] O. T. Faboya, P.-O. Siebers, B. Ryan, and G. P. Figueredo, "How do Travellers Decide: A Stochastic Modelling Approach to Determine Decision Factor Significance," in Procs of the European Modelling and Simulation Symposium, 2018.
[8] M. Bronzini, et al., "Bureau of Transportation Statistics :Transportation in the United States Highlights from 2015 Transportation Statistics Annual Report," New Jersey, 2015.

[9] Department for Transport, "National Travel Survey: England 2014," 2015.

[10] O. T. Faboya, P. Siebers, B. Ryan, and G. P. Figueredo, "A Novel Modal Shift Modelling Framework for Transport Systems," in Proc of the 13th Annual Conference of Social Simulation, 2017.

[11] J. Rasmussen, A. M. Pejtersen, and L. P. Goodstein, Cognitive systems engineering. Wiley, 1994.

[12] K. J. Vicente, Cognitive Work Analysis Toward Safe, Productive, and Healthy. New Jersey: Lawrence Erlbaum Associates, Publlishers, 1999.

[13] G. Dalton, E. de la Pena, J. M. Vassallo, and M. Acciaro, "STRIA Road Map Transport Infrastructure," 2016.

[14] European Environmental Agency, "Investment in transport infrastructure," 2016

[15] J. Smith, et al., "Clarity from above: Transport Infrastructure The commercial applications of drone technology in the road and rail sectors," 2017.

[16] Department for Transport, "Statistical Release: Domestic Road Freight Statistics, UK," 2016.

[17] G. Baxter and I. Sommerville, "Socio-technical systems: From design methods to system engineering," Int. Comput., vol. 23, no. 4-17, 2011.

[18] G. J. M. Read, et al., "Designing sociotechnical systems with cognitive work analysis: putting theory back into practice," Ergonomics, vol. 58, no. 5 , pp. 822-851, 2015.

[19] A. Bisantz and E. Roth, "Analysis of Cognitive Work," Rev. Hum. Factors Ergon., vol. 3, no. 1, pp. 1-43, 2007.

[20]D. C. Wickens, et al., An Introduction to Human Factors Engineering, 2nd ed. London: Pearson Education, inc, 2004.

[21]B. Elix and N. Naikar, "Designing safe and effective future systems: A new approach for modelling decisions in future systems with Cognitive Work Analysis," in P 8th Int Symp of the Austr Av Psy Assoc, 2008.

[22] N. Naikar, "An Examination of The Key Concepts of The Five Phases Of Cognitive," in P Human F and Ergs Soc, 2006, pp. 447-451.

[23] G. H. Jenkins, et al., Cognitive Work Analysis: Coping with Complexity. GB: Ashgate. ProQuest ebrary. Web. 10 June 2016

[24] A. M. Pejtersen and R. Fidel, "A framework for work centered evaluation and design: A case study of IR on the web," MIRA Grenoble Meet., 1998.

[25] H. A. Stoner, et al., "Applying Ecological Interface Design to the Driving Domain: The Results of an Abstraction Hierarchy Analysis," Proc. Hum. Factors Ergon. Soc. Annu. Meet., 47, 3, pp. 444-448, 2003.

[26] V. Beanland, et al., "Applying ecological interface design principles to the design of rural highway-rail grade crossing infrastructure," in Proc of the Human F and Ergs Soc, 2017, vol. 2017-Octob, pp. 1887-1891.

[27] C. Macal and M. North, "Tutorial on agent-based modelling and simulation," J. Simul., vol. 4, pp. 151-162, 2010.

[28] C. M. Macal and M. J. North, "Tutorial on agent-based modelling and simulation," J. Simul., vol. 4, no. 3, pp. 151-162, 2010.

[29] J. Schank, "Agent-BAsed Modeling: An Introduction|Agent-Based Models," 2010. [Online]. Available: http://www.agent-basedmodels.com/blog/2010/03/30/agent-based-modeling/.

[30] C. M. Macal, "Everything you need to know about agent-based modelling and simulation,” J. Simul., vol. 10, no. 2, pp. 144-156, 2016.

[31] A. P. Engelbrecht, Computational Intelligence: An Introduction, 2nd ed. Chichester, England: John Wiley \& Sons, Ltd, 2007.

[32] L. A. Zadeh, "Fuzzy Logic = Computing with Words," IEEE Trans. FUZZY Syst., vol. 4, no. 2, 1996

[33] J. M. Keller, D. Liu, and D. B. Fogel, Fundamentals of computational intelligence: neural networks, fuzzy systems, and evolutionary computation. John Wiley \& Sons, Inc, 2016.

[34] Q. Pan, C. Darabos, and J. Moore, LNCS, vol. 7246. 2012.

[35]N. A. Stanton, et al., "Following the cognitive work analysis train of thought: exploring the constraints of modal shift to rail transport," Ergonomics, vol. 56:3, no. July 2015, pp. 522-540, 2013.

[36] J. M. Mendel, Uncertainty: Rule-Based Fuzzy Logic Systems: Introduction and New Directions. NJ: Prentice Hall PTR, 2001.

[37] W. Duch, "Towards comprehensive foundations of computational intelligence," in Challenges for Computational Intelligence, Verlag Berlin Heidelberg: Springer, 2007.

[38] R. Zobbi, "Smart Mobility and services," 2017. 